\title{
ANIMAL RESEARCH PAPER \\ Pig slurry characteristics, nutrient balance and biogas production as affected by separation and acidification
}

\author{
S. G. SOMMER ${ }^{1}$, M. HJORTH ${ }^{2}$, J. J. LEAHY ${ }^{3}$, K. ZHU ${ }^{4}$, W. CHRISTEL ${ }^{4}$, C. G. SØRENSEN ${ }^{2}$ \\ AND SUTARYO \\ ${ }^{1}$ Faculty of Engineering, University of Southern Denmark, Institute of Chemical Engineering, Biotechnology and \\ Environmental Engineering, Odense M, Denmark \\ ${ }^{2}$ Department of Engineering, Faculty of Science and Technology, Aarhus University, Tjele, Denmark \\ ${ }^{3}$ Department of Chemical and Environmental Sciences, Faculty of Science and Engineering, University of Limerick, \\ Limerick, Ireland \\ ${ }^{4}$ Department of Plant and Environmental Sciences, Faculty of Science, University of Copenhagen, Frederiksberg C, \\ Denmark \\ ${ }^{5}$ Faculty of Animal and Agricultural Sciences, Diponegoro University, Semarang, Indonesia
}

(Received 11 June 2013; revised 27 January 2014; accepted 31 March 2014;

first published online 22 July 2014)

\section{SUMMARY}

Animal slurry is separated in order to avoid excessive nitrogen, phosphorus and potassium (NPK) fertilization of crops in the field. To enhance fertilizer efficiency further, slurry and its separation products may be acidified, for instance in animal houses. The current study quantified the effects of these treatments, both individually and in combination, on fertilizer efficiency, energy production and heavy metal accumulation as a result of manure management. Acidification increased the availability of $\mathrm{N}$ to plants in the manure applied, and provided a better match between plant-available NPK in the manure and separation fraction applied to fields and crop need. Total biogas production was not affected by separation, whereas acidification reduced biogas production because the process was inhibited by a low $\mathrm{pH}$ and a high sulphur concentration. The amount of copper applied per hectare in the liquid manure to the wheat field was lower than the amount taken up and more zink and copper was applied in the solid fraction to maize field than taken up. The transportation and field application of solids and liquids did not increase management costs when compared to the transportation of slurry alone, but the investment and running costs of separators and manure acidification increased overall management costs.

\section{INTRODUCTION}

Managed properly, animal slurry is an environmentally friendly source of nutrients for crop production, an amendment to improve soil and a resource for energy production. Mismanagement of slurry causes hazards to the environment in the form of greenhouse gas emissions, eutrophication of recipient waters and pollution of groundwater (Sutton et al. 2011).

Livestock farming is intensifying and becoming more specialized, leading to the decoupling of livestock farming from plant production and creating

\footnotetext{
* To whom all correspondence should be addressed. Email: sgs@ kbm.sdu.dk
}

the need to transport slurry to nutrient-deficient crop production areas. Separation of animal slurry produces a nutrient-rich solid fraction that can be transported at a low cost per unit nutrient, enabling efficient recycling. The efficiencies of the various separation techniques of different manures are not equal (Hjorth et al. 2010), making the impact of introducing manure pre-treatments difficult to predict.

Acidification has proved to be an efficient method for reducing ammonia $\left(\mathrm{NH}_{3}\right)$ emissions (Kai et al. 2008). The hypothesis advanced in current article is that the combined effect of separation and acidification may be beneficial in terms of controlling 
$\mathrm{NH}_{3}$ emissions, as well as contributing to improved plant nutrient use.

There is a need to quantify the effects of an acidification pre-treatment on slurry characteristics and nutrient separation efficiencies, as well as its effects on the assessment of energy production, heavy metal accumulation and fertilizer efficiencies of the manure products. The current study provides insights into how an acidification pre-treatment before slurry separation affects the composition of separation products, with a focus on organic matter, plant nutrients, energy and heavy metal accumulation.

\section{MATERIALS AND METHODS}

During the spring of 2012, slurry from two separate pig pens - each housing 32 growing-finishing pigs - at the Grønhøj research station, Denmark $\left(56^{\circ} 38^{\prime} \mathrm{N}\right.$, $9^{\circ} 18^{\prime} \mathrm{E}$ ) was collected and stored separately in $20 \mathrm{~m}^{3}$ tanks. The slurry in the channels of one of the sections was acidified with sulphuric acid $\left(\mathrm{H}_{2} \mathrm{SO}_{4}\right)$ to $\mathrm{pH} 5 \cdot 5$ on a daily basis, whereas the slurry in the other section was left untreated. The fattening pig house was managed using Danish standard production guidelines for housing, feeding and management conditions. The untreated pig slurry and continuously acidified pig slurry were stirred prior to full-scale separation at the pig farm by means of three different separation technologies: (i) a screw press (Börger BS50, Börger $\mathrm{GmbH}$, Borken-Weseke, Germany), (ii) flocculation using polymers and drainage with a filter band separator (AL-2 model 812P, Hovborg, Denmark) and (iii) a decanter centrifuge (Pieralisi, Gruppo Pieralisi-MAIP S.p.A., Jesi (AN) Italy). The polymers used in the drainage study were Praestol K133L (Demols Inc., Houston, Texas, USA) with a medium cationic charge (c. 60\%) for untreated slurry, and Praestol K144L with a high cationic charge (c. 75\%) for acidified slurry. The flows of slurry and separation products were documented. The inorganic and organic components, particle size and energy content of the untreated non-acidified and acidified slurries, as well as those of their separation products, were determined. Two samples of each slurry and separation category were collected, stored at $-18{ }^{\circ} \mathrm{C}$ and, after thawing, analysed in the laboratory.

The slurry $\mathrm{pH}$ was determined on-site using a standard $\mathrm{pH}$ electrode (Radiometer, Copenhagen, Denmark). Total ammoniacal nitrogen (TAN $=\mathrm{NH}_{3}+$ ammonium $\left.\left(\mathrm{NH}_{4}^{+}\right)\right)$and total Kjeldahl nitrogen (Total-N) were determined according to standard procedures using a Kjeltec 2011 instrument (Foss, Höganäs, Sweden) according to the procedures given by APHA (2005). The total phosphorus (total-P), potassium $(\mathrm{K})$, sulphur $(\mathrm{S})$, copper $(\mathrm{Cu})$ and zinc $(\mathrm{Zn})$ content of the slurry and the solid and liquid fractions were determined by inductively coupled plasma optical emission spectrometry (ICP-OES, ICAP 6300 DUO Thermo Scientific, Germany) according to the procedures given in European Regulation 2009/ 152/EC (EC 2009).

Water-soluble $P$ was determined by three successive extractions at a solid-liquid mass ratio of $1: 25 \mathrm{~g} / \mathrm{g}$ followed by centrifugation steps $(10000 \mathrm{rpm}$ for $10 \mathrm{~min}$ ) and decanting of the supernatant after each centrifugation treatment. Following extraction, the concentration of water-soluble $\mathrm{P}$ was determined using a spectrophotometer (Helios a, Fisher Scientific, Loughborough, UK) according to the procedures provided by Murphy \& Riley (1962). Total dihydrogen sulphide $\left(\mathrm{H}_{2} \mathrm{~S}_{\text {tot }}=\right.$ dihydrogen sulphide $\left(\mathrm{H}_{2} \mathrm{~S}\right)+$ hydrogen sulphide $\left(\mathrm{HS}^{-}\right)+$sulphide $\left(\mathrm{S}_{2}^{-}\right)$was determined by precipitation with zinc, $\mathrm{H}_{2} \mathrm{~S}$ gas capture, colour reaction and spectrometric quantification using a spectrophotometer (Helios a, Fisher Scientific, Loughborough, UK) according to the procedures given by Eriksen et al. (2010).

The dry matter (DM) concentration of the manures was determined gravimetrically after a $24 \mathrm{~h}$ drying period at $103^{\circ} \mathrm{C}$ in accordance with the Danish Standard DS/EN 14346 (Anonymous 2007). The ash content was determined following combustion at $550{ }^{\circ} \mathrm{C}$ for $2 \mathrm{~h}$. Measurements were carried out in triplicate. Volatile solids (VS) were calculated as the difference between the DM and ash content of the sample.

Total inorganic carbon $\quad(\mathrm{TIC}=$ carbon dioxide $\left(\mathrm{CO}_{2}\right)+$ bicarbonate $\left(\mathrm{HCO}_{3}^{-}\right)+$carbonate $\left.\left(\mathrm{CO}_{3}^{2-}\right)\right)$ was determined in a closed system consisting of two glass flasks connected by glass tubes (Sommer \& Husted 1995). One flask contained the slurry samples and the other contained an absorbent of $0 \cdot 2 \mathrm{M}$ sodium hydroxide $(\mathrm{NaOH})$. Hydrochloric acid $(1 \mathrm{M}$ $\mathrm{HCl}$ ) was added to the slurry in amounts exceeding the content of the base components, and the evolved $\mathrm{CO}_{2}$ collected in the absorbent. Subsequently, $1 \mathrm{M}$ barium chloride $\left(\mathrm{BaCl}_{2}\right)$ was added to the absorbent and TIC was determined by titration of the absorbent with $0.100 \mathrm{M} \mathrm{HCl}$ using phenolphthalein as an indicator.

Determination of the specific gravity of the manure and separation products (uncompacted) was 
performed in duplicate by weighing the content of a $1000 \mathrm{ml}$ volumetric flask to the nearest $10 \mathrm{mg}$.

Volatile fatty acids (VFA, C2-C5 organic acids) were measured using a gas chromatograph (Agilent 7890A, Santa Clara, California, USA) with a HP-INNOWax, $3 \mathrm{~m} \times 0.25 \mathrm{~mm} \times 0 \cdot 25 \mu \mathrm{m}$ column and flame ionization detector. The carrier gas was helium. The temperature of the column was gradually increased from 110 to $220^{\circ} \mathrm{C}$ at $10^{\circ} \mathrm{C} / \mathrm{min}$.

Hemicelluloses, cellulose and lignin were determined in accordance with Van Soest's protocol for fibre analysis (Van Soest 1963; Goering \& Van Soest 1970) using a Fibertec 2010 instrument (Foss, Höganäs, Sweden). Neutral detergent fibre (NDF) was determined according to Mertens (2002). Acid detergent fibre (ADF) and acid detergent lignin (ADL) were determined according to ISO standards (Van Soest 1963).

The biochemical methane potential (BMP) of the manure and solid manure fractions were determined by means of a batch technique using 500-ml infusion bottles (Møller et al. 2004a). Inoculum from mesophilic secondary biogas storage was added to all samples on a $1: 1$ ratio on the basis of the volatile solid content. The headspace was 150-ml. Blanks were tested using $200 \mathrm{~g}$ of inoculum to correct gas production. After adding the substrate inoculum mixture, the digesters were closed with butyl rubber stoppers, sealed with aluminium crimps, flushed with $\mathrm{N}_{2}$ atmosphere and incubated at $37( \pm 0 \cdot 5){ }^{\circ} \mathrm{C}$. All assays were performed in triplicate. Gas volume was measured for 90 days on a regular basis, either by replacing water or using a large syringe. Methane $\left(\mathrm{CH}_{4}\right)$ and $\mathrm{CO}_{2}$ were determined simultaneously by a gas chromatograph (Agilent 7890A, Santa Clara, California, USA) equipped with a thermal conductivity detector and an Alltech CTR $30 \mathrm{~m} \times 0.320 \mathrm{~mm}$ column. The carrier gas was helium $(300 \mathrm{~mm} / \mathrm{s})$ and the injection volume was $0.4 \mathrm{ml}$. The injector temperature was $110^{\circ} \mathrm{C}$ and the detector and oven temperature was $250^{\circ} \mathrm{C}$.

\section{Calculations}

Using data from the Van Soest analysis, the cell wall fraction of the VS in the slurry was assessed as follows: NDF is composed of hemicellulose, cellulose and lignin, and the components that are dissolved when determining NDF are proteins, fats and carbohydrates; ADF is composed of cellulose and lignin; hemicellulose is equal to the difference between NDF and ADF. Acid detergent lignin is composed of lignin, and cellulose is calculated as the difference between ADF and ADL.

The decision was taken to use $30 \mathrm{t}$ of acidified or of untreated slurry, removed from the pig house as the base unit when assessing the field acreage that the slurry or separation fractions could support with: (A) plant-available $\mathrm{N}$ to fulfil the crop need or (B) the acreage of fields for which application rate of the slurry and slurry separation fractions could fulfil the plant need for $\mathrm{N}, \mathrm{P}$ or $\mathrm{K}$ at the lowest manure application rate. The amount of $\mathrm{N}, \mathrm{P}$ and $\mathrm{K}$ in the slurry or separation fractions was calculated by estimating losses of $\mathrm{N}$ during storage and application, and transfer of $\mathrm{N}, \mathrm{P}$ and $\mathrm{K}$ from solid manure storage to liquid storage in leaking water (Table 1). A mass balance was calculated of the components in the slurry and separation fractions during the management chain from removal until field application. The total N, P and $\mathrm{K}$ multiplied by the corresponding mineral fertilizer equivalent (MFE; Table 1) and divided by the crop fertilizer requirement (Table 2) gave the area of crops whose nutrient need can be met by the application of slurry and slurry fractions. This area differs between $\mathrm{N}, \mathrm{P}$ and $\mathrm{K}$ by manure category and crop. It was therefore decided to present the acreage required based on efficient $\mathrm{N}, \mathrm{P}$ or $\mathrm{K}$ use when applying the slurry and liquid fractions to fulfil the fertilizer need of wheat, and the solid manure fraction to fulfil the fertilizer need of maize.

The contents of $\mathrm{Cu}$ and $\mathrm{Zn}$ from the applied slurry or slurry fractions were compared with the amount removed by the harvested crops. As regards the removal of $\mathrm{Cu}$ and $\mathrm{Zn}$ with harvested wheat and maize, the following rationale was used: irrespective of the manure application rate, c. 0.01-0.12 kg Cu/ha can be removed in the grain of wheat plants and $0 \cdot 02-0 \cdot 10 \mathrm{~kg} \mathrm{Cu} / \mathrm{ha}$ with the wheat straw (Cabral et al. 1998). Harvesting of maize grain and straw may remove 0.03-0.06 kg Cu/ha, based on the $\mathrm{Cu}$ concentrations in the whole maize plant grown on soil amended with pig manure (Berenguer et al. 2008).

The amount of $\mathrm{Zn}$ removed from wheat grain and straw is $0 \cdot 05-0 \cdot 16$ and $0 \cdot 10-0 \cdot 37 \mathrm{~kg} \mathrm{Zn} / \mathrm{ha}$, respectively, for pig manure application rates between 5 and $25 \mathrm{t} /$ ha (Cabral et al. 1998). Based on a Zn content of between 13.4 and $17.5 \mathrm{mg} \mathrm{Zn/kg}$ in maize biomass (Berenguer et al. 2008), 0.30-0.47 kg Zn/ha can be removed from the whole maize plant at pig manure application rates of 29-51 t/ha.

The operating costs for the different separation techniques were based on conventional methods for 
Table 1. Total gaseous emission of $\mathrm{NH}_{3}, \mathrm{~N}_{2}$ and $\mathrm{N}_{2} \mathrm{O}$ during storage of liquid manure with cover and solid manure without cover (Hansen et al. 2006, 2008). Leaching of nitrogen (N), phosphorus $(P)$ and potassium (K) from solid manure stores (Sommer 2001). The emission of $N$ gases from acidified solid manure is a qualified estimate. Mineral fertilizer equivalent of solid manure applied to maize fields and of slurry and liquid manure applied to winter wheat (Birkmose 2009; Sørensen \& Eriksen 2009; Jensen 2013). No leaching is expected from slurry and liquid manure stored in concrete slurry tanks, and liquid leaching from solid manure is pumped into the liquid manure store

\begin{tabular}{|c|c|c|c|c|c|}
\hline & \multirow{2}{*}{$\begin{array}{l}\text { Gaseous } \\
\text { emissions } \\
\mathrm{N} \text { (proportion } \\
\text { of total } \mathrm{N})\end{array}$} & \multicolumn{3}{|c|}{ Leaching from store } & \multirow{2}{*}{$\begin{array}{l}\text { MFE* } \\
\mathrm{N} \text { (proportion } \\
\text { of total } \mathrm{N})\end{array}$} \\
\hline & & $\begin{array}{l}\mathrm{N} \text { (proportion } \\
\text { of total } \mathrm{N})\end{array}$ & $\begin{array}{l}\mathrm{P}(\text { proportion } \\
\text { of total } \mathrm{P})\end{array}$ & $\begin{array}{l}\mathrm{K} \text { (proportion } \\
\text { of total } \mathrm{K} \text { ) }\end{array}$ & \\
\hline $\begin{array}{l}\text { Solid fraction (screw press or decanter } \\
\text { centrifuge) }\end{array}$ & $0 \cdot 40$ & $0 \cdot 02$ & $0 \cdot 02$ & $0 \cdot 12$ & $0 \cdot 20$ \\
\hline Solid fraction (flocculation and drainage) & $0 \cdot 15$ & $0 \cdot 02$ & $0 \cdot 02$ & $0 \cdot 12$ & $0 \cdot 20$ \\
\hline Solid fraction (all separators, acidified) & $0 \cdot 05$ & $0 \cdot 02$ & $0 \cdot 02$ & $0 \cdot 12$ & $0 \cdot 30$ \\
\hline Slurry (pig) & $0 \cdot 02$ & $0 \cdot 00$ & $0 \cdot 00$ & $0 \cdot 00$ & $0 \cdot 65$ \\
\hline Slurry (pig, acidified) & 0 & 0 & 0 & 0 & $0 \cdot 80$ \\
\hline Liquid fraction & 2 & 0 & 0 & 0 & $0 \cdot 80$ \\
\hline Liquid fraction (acidified) & 0 & 0 & 0 & 0 & $0 \cdot 90$ \\
\hline Biogas digestate & 2 & 0 & 0 & 0 & $0 \cdot 70$ \\
\hline
\end{tabular}

* Availability of N, P and K is expressed as MFE, which quantifies the amount of fertilizers $\mathrm{N}, \mathrm{P}$ and $\mathrm{K}$ that is substituted by $\mathrm{N}$, $\mathrm{P}$ and $\mathrm{K}$ in the manure (fraction). For $\mathrm{P}$ and $\mathrm{K}$, the long-term MFE is 1.00 and for $\mathrm{N}$, depending on the slurry properties and separation technique, MFE is between $0 \cdot 20$ and 0.90 of total $N$.

Table 2. Danish norms for the maximum addition of fertilizer to crops (information from L. Knudsen, Danish Knowledge Centre for Agriculture, 2013)

\begin{tabular}{llllrr}
\hline \hline & & & \multicolumn{2}{c}{ Crop mineral fertilizer need (kg/ha) } \\
\cline { 4 - 6 } & & $\begin{array}{l}\text { Harvest yield } \\
(\mathrm{DM} \text { kg/ha) }\end{array}$ & $\mathrm{N}$ & $\mathrm{P}$ & $\mathrm{K}$ \\
\hline Maize for silage & Whole crop & 11700 & 170 & 30 & 120 \\
Winter wheat & Grain & 6375 & 180 & 24 & 69 \\
Winter wheat & Straw & 2550 & & 15 \\
\hline \hline
\end{tabular}

DM, dry matter; $N$, nitrogen; $\mathrm{P}$, phosphorus; $\mathrm{K}$, potassium; $\mathrm{Mg}$, magnesium.

estimating the interest, depreciation and maintenance of machinery (e.g. Sørensen \& Møller 2006). The average total annual costs were given by the following equation as a combination of capital costs and variable costs:

$$
\begin{aligned}
C= & I \times\left(\left(\frac{i \times 100 \times(1-(1-w))^{n}}{1-(1+i)^{-n}}\right)\right. \\
& \left.+\left(100 \times(1-w)^{n} \times i\right)\right) \\
& +(A \times i)+(O \times(a \times f))+u+h
\end{aligned}
$$

where $C$ is the total annual cost, I the initial investment (US\$), $i$ the annual interest rate $(\% / 100), n$ the number of years over which the machine will be depreciated, $w$ the annual depreciation rate $(\% / 100), A$ the estimated labour requirement (h), I the labour cost (US\$/h), $O$ the nominal operating time $(h)$, a the maintenance cost (US\$/h), $f$ the fuel cost (US\$/h), $u$ the yearly machine insurance premium (US\$) and $h$ the yearly housing cost (US\$) (Table 3). The cost was estimated assuming that standard equipment was used to manage the slurry and slurry fractions, i.e. slurry and liquid fraction management entailed a tanker for transport and trailing hoses for the application, and solid fraction transport and application involved a muck spreader. The cost was based on normative unit costs per tonne (Farmtal Online 2013), indicating US $\$ 3.19$ for slurry/liquid fraction application and US\$3.89 for solid manure application. The application 


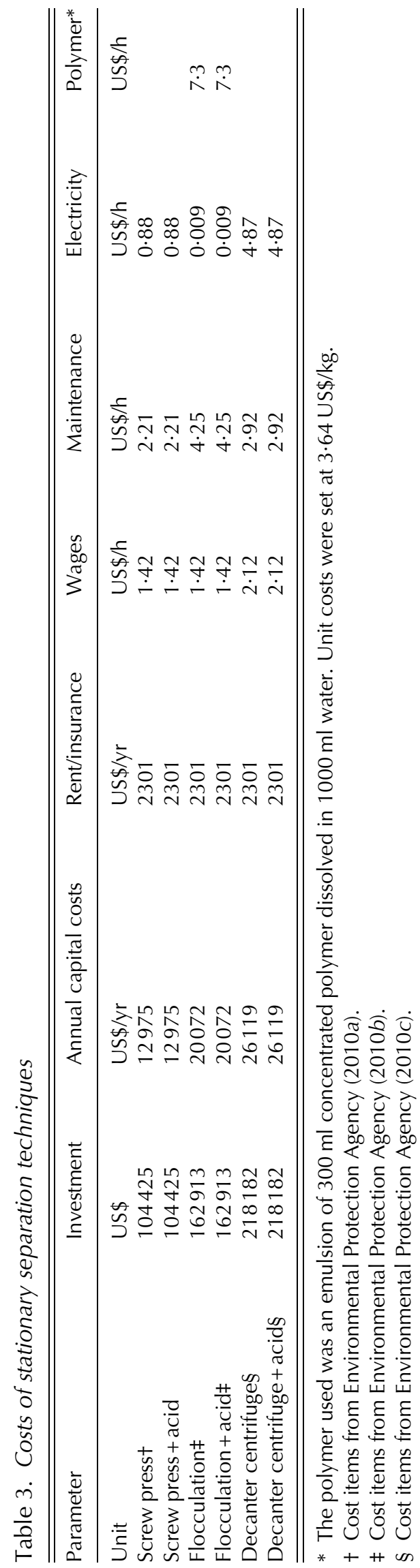

scenario involved 392 animal units (11 694 fattening pigs) producing $6899 \mathrm{t}$ of raw slurry per year, and application rates were calculated using the NPK requirements given in Table 2 . The stipulated configuration of the assumed application area involved quarter circles radiating from the location of the storage facilities, indicating an average transport distance of three quarters of the radius of the quarter circle. The costs of acidifying $1 \mathrm{t}$ of slurry were calculated using the following input data: investment US $\$ 0 \cdot 22$ million (US $\$ 0 \cdot 15-0 \cdot 27$ million) and acid cost US\$0.19/( $\left.\mathrm{H}_{2} \mathrm{SO}_{4}\right)$.

With regard to statistics, the BMP of each slurry and slurry fraction was measured in triplicate and comparisons were performed with Tukey's hoest significant difference test. In the current full-scale farm experiment, it was not possible to obtain replicate or triplicate measurements of a treatment due to management challenges, time restrictions and access to facilities. Therefore, statistical analyses were not performed on treatment effects.

\section{RESULTS}

The DM concentration of the pig slurry was c. $90 \mathrm{~g} / \mathrm{kg}$ (Table 4), which is relatively high for pig slurry collected in Denmark. Consequently, the slurry had higher concentrations of most of the components (i.e. the concentrations of $\mathrm{K}$ and total- $\mathrm{N}$ were c. 1.5 and two times higher, respectively) than the concentrations measured in the study of Sommer \& Husted (1995), while the VFA contents of untreated and acid-treated slurry (Table 5) were about five and three times greater. The hydrogen sulphide concentration was below $0.007 \mathrm{mg} / \mathrm{kg}$ for both acidified and non-acidified manure. In contrast, ortho-P was higher in the acidified slurry than in the untreated slurry (Table 6).

Acidification of slurry interfered with the separation, thus all three separators produced less solid fraction when using acidified slurry (Fig. 1(a)). The DM concentration in the solid fractions was not affected by slurry acidification (Fig. 1(b)).

The non-acidified slurry and non-acidified liquid fraction from the screw press and decanter centrifuge showed a higher BMP/kg VS than the acidified slurry, acidified liquid fractions and solid fractions (Fig. 2(a)). The largest BMP/kg VS of the liquid fractions was from the decanter centrifuge and the screw press. The liquid fractions from flocculation and drainage were smaller than from all the other fractions and slurry. In contrast, the solid fraction from flocculation and drainage 
Table 4. Content of inorganic components and density of the slurry and the liquid and solid fractions included in the study (sD in parentheses, $n=2)$

\begin{tabular}{|c|c|c|c|c|c|c|c|c|c|c|c|c|c|}
\hline Treatment & Acid & Fraction & $\begin{array}{l}\text { DM } \\
(\mathrm{g} / \mathrm{kg})\end{array}$ & $\begin{array}{l}\text { Ash } \\
(\mathrm{g} / \mathrm{kg})\end{array}$ & $\begin{array}{l}\text { Density } \\
(\mathrm{kg} / \mathrm{l})\end{array}$ & $\mathrm{pH}$ & $\begin{array}{l}\text { Total N } \\
(\mathrm{g} / \mathrm{kg})\end{array}$ & $\begin{array}{l}\text { TAN } \\
(\mathrm{g} / \mathrm{kg})\end{array}$ & $\begin{array}{l}P \\
(\mathrm{~g} / \mathrm{kg})\end{array}$ & $\begin{array}{l}\mathrm{K} \\
(\mathrm{g} / \mathrm{kg})\end{array}$ & $\begin{array}{l}\mathrm{S} \\
(\mathrm{g} / \mathrm{kg})\end{array}$ & $\begin{array}{l}\mathrm{Cu} \\
(\mathrm{mg} / \mathrm{kg})\end{array}$ & $\begin{array}{l}\mathrm{Zn} \\
(\mathrm{mg} / \mathrm{kg})\end{array}$ \\
\hline Untreated & + & Slurry house & 90 & 20 & $1 \cdot 04$ & $5 \cdot 6$ & $5 \cdot 7$ & $3 \cdot 8$ & $1 \cdot 4$ & $2 \cdot 7$ & $3 \cdot 3$ & $12 \cdot 5$ & $65 \cdot 5$ \\
\hline Untreated & + & Slurry house & $(3 \cdot 8)$ & $(0 \cdot 7)$ & & $(0 \cdot 10)$ & $(0 \cdot 01)$ & $(0 \cdot 00)$ & $(0 \cdot 00)$ & $(0 \cdot 00)$ & $(0 \cdot 07)$ & $(0 \cdot 71)$ & $(0 \cdot 71)$ \\
\hline Untreated & + & Slurry store & 84 & 21 & N.D. & $5 \cdot 3$ & $6 \cdot 3$ & $3 \cdot 9$ & $1 \cdot 4$ & $3 \cdot 0$ & $4 \cdot 3$ & $10 \cdot 8$ & $55 \cdot 8$ \\
\hline Untreated & + & Slurry store & $(1 \cdot 2)$ & $(1 \cdot 0)$ & & $(0 \cdot 07)$ & $(0 \cdot 04)$ & $(0 \cdot 20)$ & $(0 \cdot 05)$ & $(0 \cdot 10)$ & $(0 \cdot 21)$ & $(0 \cdot 50)$ & $(2 \cdot 50)$ \\
\hline Untreated & & Slurry & 83 & 18 & N.D. & $6 \cdot 7$ & $6 \cdot 3$ & $4 \cdot 3$ & $1 \cdot 6$ & $3 \cdot 1$ & 0.6 & $12 \cdot 8$ & $62 \cdot 3$ \\
\hline Untreated & & Slurry & $(2 \cdot 8)$ & $(0 \cdot 5)$ & & $(0 \cdot 17)$ & $(0 \cdot 05)$ & $(0 \cdot 20)$ & $(0 \cdot 05)$ & $(0 \cdot 08)$ & $(0 \cdot 02)$ & $(0.96)$ & $(2 \cdot 99)$ \\
\hline Untreated & & Slurry & 95 & $21 \cdot 00$ & N.D. & $7 \cdot 0$ & $7 \cdot 8$ & $5 \cdot 8$ & 1.9 & $3 \cdot 6$ & 0.6 & $13 \cdot 3$ & $79 \cdot 3$ \\
\hline Untreated & & Slurry & $(4 \cdot 5)$ & $(0 \cdot 8)$ & & $(0 \cdot 10)$ & $(0 \cdot 30)$ & $(0 \cdot 35)$ & $(0 \cdot 10)$ & $(0 \cdot 12)$ & $(0 \cdot 02)$ & $(0.96)$ & $(2 \cdot 50)$ \\
\hline Screw press & & Liquid & 66 & 21 & $1 \cdot 04$ & $7 \cdot 0$ & $7 \cdot 5$ & $5 \cdot 5$ & $2 \cdot 0$ & $3 \cdot 5$ & $0 \cdot 5$ & $14 \cdot 0$ & $83 \cdot 5$ \\
\hline Screw press & & Liquid & $(0 \cdot 7)$ & $(0 \cdot 7)$ & & $(0 \cdot 06)$ & $(0 \cdot 02)$ & $(0 \cdot 06)$ & $(0 \cdot 07)$ & $(0 \cdot 14)$ & $(0 \cdot 01)$ & $(0 \cdot 00)$ & $(0 \cdot 71)$ \\
\hline Screw press & & Fibre & 271 & 26 & $0 \cdot 34$ & $7 \cdot 9$ & $7 \cdot 4$ & $4 \cdot 5$ & $2 \cdot 4$ & $3 \cdot 2$ & $1 \cdot 1$ & $12 \cdot 5$ & $71 \cdot 5$ \\
\hline Screw press & & Fibre & $(5 \cdot 9)$ & $(0 \cdot 7)$ & & $(0 \cdot 08)$ & $(0 \cdot 11)$ & $(0 \cdot 40)$ & $(0 \cdot 07)$ & $(0 \cdot 00)$ & $(0 \cdot 00)$ & $(0 \cdot 71)$ & $(2 \cdot 12)$ \\
\hline Screw press & + & Liquid & 67 & 22 & $1 \cdot 02$ & $5 \cdot 4$ & $6 \cdot 3$ & $4 \cdot 2$ & $1 \cdot 4$ & $3 \cdot 0$ & $4 \cdot 3$ & $10 \cdot 0$ & $56 \cdot 5$ \\
\hline Screw press & + & Liquid & $(0 \cdot 4)$ & $(0 \cdot 7)$ & & $(0 \cdot 04)$ & $(0 \cdot 01)$ & $(0 \cdot 00)$ & $(0 \cdot 00)$ & $(0 \cdot 14)$ & $(0 \cdot 64)$ & $(0 \cdot 00)$ & $(0 \cdot 71)$ \\
\hline Screw press & + & Fibre & 247 & 21 & $0 \cdot 42$ & $5 \cdot 4$ & $6 \cdot 4$ & $3 \cdot 5$ & $1 \cdot 5$ & $2 \cdot 6$ & $4 \cdot 0$ & $10 \cdot 4$ & $54 \cdot 5$ \\
\hline Screw press & + & Fibre & $(6 \cdot 0)$ & $(0 \cdot 7)$ & & $(0 \cdot 06)$ & $(0 \cdot 33)$ & $(0 \cdot 11)$ & $(0 \cdot 07)$ & $(0 \cdot 00)$ & $(0 \cdot 14)$ & $(0 \cdot 85)$ & $(6 \cdot 36)$ \\
\hline Flocculation-drainage & & Liquid & 9 & 3 & $1 \cdot 00$ & $7 \cdot 1$ & $2 \cdot 1$ & $1 \cdot 9$ & $0 \cdot 2$ & $1 \cdot 4$ & $0 \cdot 1$ & $<0 \cdot 01$ & $1 \cdot 9$ \\
\hline Flocculation-drainage & & Liquid & $(0 \cdot 6)$ & $(1 \cdot 4)$ & & $(0 \cdot 08)$ & $(0 \cdot 00)$ & $(0 \cdot 02)$ & $(0 \cdot 00)$ & $(0 \cdot 00)$ & $(0 \cdot 01)$ & & $(0 \cdot 14)$ \\
\hline Flocculation-drainage & & Fibre & 127 & 24 & $0 \cdot 91$ & $7 \cdot 0$ & $7 \cdot 6$ & $4 \cdot 8$ & $2 \cdot 5$ & $2 \cdot 8$ & $0 \cdot 7$ & $18 \cdot 5$ & $110 \cdot 0$ \\
\hline Flocculation-drainage & & Fibre & $(8 \cdot 0)$ & $(1 \cdot 4)$ & & $(0 \cdot 03)$ & $(0 \cdot 32)$ & $(0 \cdot 26)$ & $(0 \cdot 00)$ & $(0 \cdot 14)$ & $(0 \cdot 03)$ & $(0 \cdot 71)$ & $(0 \cdot 00)$ \\
\hline Flocculation-drainage & + & Liquid & 21 & 10 & $1 \cdot 01$ & $5 \cdot 1$ & $2 \cdot 7$ & $2 \cdot 2$ & 0.6 & $1 \cdot 7$ & $2 \cdot 4$ & $<0 \cdot 01$ & $1 \cdot 1$ \\
\hline Flocculation-drainage & + & Liquid & $(0 \cdot 4)$ & $(0 \cdot 0)$ & & $(0 \cdot 26)$ & $(0 \cdot 01)$ & $(0 \cdot 00)$ & $(0 \cdot 01)$ & $(0 \cdot 07)$ & $(0 \cdot 07)$ & & \\
\hline Flocculation-drainage & + & Fibre & 123 & 21 & $0 \cdot 98$ & $5 \cdot 4$ & $6 \cdot 6$ & $3 \cdot 2$ & $1 \cdot 4$ & $2 \cdot 4$ & $3 \cdot 7$ & $18 \cdot 5$ & $105 \cdot 0$ \\
\hline Flocculation-drainage & + & Fibre & $(6 \cdot 6)$ & $(0 \cdot 7)$ & & $(0 \cdot 07)$ & $(0 \cdot 23)$ & $(0 \cdot 21)$ & $(0 \cdot 00)$ & $(0 \cdot 00)$ & $(0 \cdot 07)$ & $(0 \cdot 71)$ & $(7 \cdot 07)$ \\
\hline Decanter centrifuge & & Liquid & 43 & 13 & $1 \cdot 02$ & $6 \cdot 5$ & $6 \cdot 8$ & $5 \cdot 1$ & $0 \cdot 5$ & $3 \cdot 6$ & $0 \cdot 5$ & $13 \cdot 0$ & $68 \cdot 5$ \\
\hline Decanter centrifuge & & Liquid & $(0 \cdot 2)$ & $(0 \cdot 0)$ & & $(1 \cdot 10)$ & $(0 \cdot 01)$ & $(0 \cdot 09)$ & $(0 \cdot 00)$ & $(0 \cdot 07)$ & $(0 \cdot 01)$ & $(0 \cdot 00)$ & $(0 \cdot 71)$ \\
\hline Decanter centrifuge & & Fibre & 332 & 52 & $0 \cdot 32$ & $7 \cdot 6$ & $9 \cdot 5$ & $6 \cdot 0$ & $7 \cdot 3$ & $3 \cdot 1$ & $1 \cdot 6$ & $17 \cdot 0$ & $135 \cdot 0$ \\
\hline Decanter centrifuge & & Fibre & $(5 \cdot 9)$ & $(2 \cdot 8)$ & & $(0 \cdot 05)$ & $(0 \cdot 30)$ & $(0 \cdot 26)$ & $(0 \cdot 64)$ & $(0 \cdot 07)$ & $(0 \cdot 00)$ & $(1 \cdot 41)$ & $(7 \cdot 07)$ \\
\hline Decanter centrifuge & + & Liquid & 54 & 19 & $1 \cdot 03$ & $5 \cdot 4$ & $6 \cdot 0$ & $4 \cdot 1$ & $1 \cdot 2$ & $3 \cdot 0$ & $4 \cdot 5$ & $9 \cdot 3$ & $45 \cdot 0$ \\
\hline Decanter centrifuge & + & Liquid & $(2 \cdot 0)$ & $(0 \cdot 0)$ & & $(0 \cdot 08)$ & $(0 \cdot 01)$ & $(0 \cdot 00)$ & $(0 \cdot 00)$ & $(0 \cdot 07)$ & $(0 \cdot 07)$ & $(0.99)$ & $(2 \cdot 83)$ \\
\hline Decanter centrifuge & + & Fibre & 327 & 32 & $0 \cdot 31$ & $5 \cdot 5$ & $8 \cdot 0$ & $3 \cdot 4$ & $2 \cdot 5$ & $2 \cdot 3$ & $3 \cdot 9$ & $19 \cdot 5$ & $145 \cdot 0$ \\
\hline Decanter centrifuge & + & Fibre & $(4 \cdot 0)$ & $(0 \cdot 0)$ & & $(0 \cdot 07)$ & $(0 \cdot 01)$ & $(0 \cdot 00)$ & $(0 \cdot 07)$ & $(0 \cdot 00)$ & $(0 \cdot 14)$ & $(0 \cdot 71)$ & $(7 \cdot 07)$ \\
\hline
\end{tabular}

DM: dry matter; N: nitrogen; TAN: total ammoniacal nitrogen; P: phosphorus; K: potassium; S: sulphur; Cu: copper; Zn: zinc. 
Table 5. Content of organic components in the slurry and liquid and solid fractions studied (SD in parentheses, $n=2$ ). VFA: volatile fatty acids; TIC: total inorganic carbon

\begin{tabular}{|c|c|c|c|c|c|c|c|c|c|c|c|c|c|c|}
\hline Treatment & Acid & Fraction & & $\begin{array}{l}\text { Acetic } \\
\text { acid } \\
(\mathrm{mg} / \mathrm{l})\end{array}$ & $\begin{array}{l}\text { Propionic } \\
\text { acid } \\
(\mathrm{mg} / \mathrm{l})\end{array}$ & $\begin{array}{l}\text { Iso } \\
\text { butanoic } \\
\text { acid } \\
(\mathrm{mg} / \mathrm{l})\end{array}$ & $\begin{array}{l}\text { Butanoic } \\
\text { acid } \\
(\mathrm{mg} / \mathrm{l})\end{array}$ & $\begin{array}{l}\text { Iso } \\
\text { valeric } \\
\text { acid } \\
(\mathrm{mg} / \mathrm{l})\end{array}$ & $\begin{array}{l}\text { Valeric } \\
\text { acid } \\
(\mathrm{mg} / \mathrm{l})\end{array}$ & $\begin{array}{l}\text { VFA } \\
(\mathrm{mol} / \mathrm{kg})\end{array}$ & $\begin{array}{l}\mathrm{TIC} \\
(\mathrm{mol} / \mathrm{kg})\end{array}$ & $\begin{array}{l}\text { Lignin } \\
(\mathrm{g} / \mathrm{kg})\end{array}$ & $\begin{array}{l}\text { Hemi- } \\
\text { cellulose } \\
(\mathrm{g} / \mathrm{kg})\end{array}$ & $\begin{array}{l}\text { Cellulose } \\
(\mathrm{g} / \mathrm{kg})\end{array}$ \\
\hline Untreated & + & Slurry & Avg.* & 5035 & 2199 & 265 & 3266 & 487 & 693 & 11946 & $0 \cdot 02$ & 80 & 128 & 160 \\
\hline Untreated & + & Slurry & SD & & & & & & & & & (2) & (11) & (10) \\
\hline Untreated & + & Slurry & Avg. & 5035 & 2199 & 264 & 3265 & 487 & 693 & 11946 & $0 \cdot 01$ & 67 & 122 & 110 \\
\hline Untreated & + & Slurry & SD & & & & & & & & & (7) & (1) & (23) \\
\hline Untreated & & Slurry & Avg. & 9843 & 3588 & 863 & 2881 & 1154 & 390 & 18722 & $0 \cdot 01$ & 79 & 178 & 132 \\
\hline Untreated & & Slurry & SD & & & & & & & & & (7) & $(0 \cdot 3)$ & (4) \\
\hline Untreated & & Slurry & Avg. & & & & & & & & $0 \cdot 01$ & 91 & 183 & 168 \\
\hline Untreated & & Slurry & SD & & & & & & & & & (2) & (2) & (4) \\
\hline Screw press & & Liquid & Avg. & 10024 & 3689 & 888 & 2955 & 1206 & 414 & 19177 & $0 \cdot 10$ & 56 & 76 & 49 \\
\hline Screw press & & Liquid & $\mathrm{SD}$ & & & & & & & & & (4) & (15) & (9) \\
\hline Screw press & & Fibre & Avg. & 9039 & 3051 & 688 & 1904 & 977 & 304 & 15963 & $0 \cdot 10$ & 136 & 306 & 299 \\
\hline Screw press & & Fibre & SD & & & & & & & & & (1) & (18) & (9) \\
\hline Screw press & + & Liquid & Avg. & 5170 & 2260 & 271 & 3334 & 496 & 711 & 12242 & $0 \cdot 10$ & 28 & 57 & 47 \\
\hline Screw press & + & Liquid & SD & & & & & & & & & (2) & (13) & (7) \\
\hline Screw press & + & Fibre & Avg. & 4308 & 1764 & 205 & 2618 & 382 & 564 & 9841 & $0 \cdot 17$ & 129 & 339 & 297 \\
\hline Screw press & + & Fibre & SD & & & & & & & & & (4) & (12) & (7) \\
\hline Flocculation-drainage & & Liquid & Avg. & 3754 & 1347 & 311 & 1070 & 423 & 152 & 7057 & $0 \cdot 13$ & 10 & $0 \cdot 0$ & 22 \\
\hline Flocculation-drainage & & Liquid & SD & & & & & & & & & (3) & (7) & (7) \\
\hline Flocculation-drainage & & Fibre & Avg. & 8717 & 3225 & 795 & 2532 & 1071 & 324 & 16664 & $0 \cdot 15$ & 100 & 178 & 167 \\
\hline Flocculation-drainage & & Fibre & SD & & & & & & & & & (6) & (9) & (10) \\
\hline Flocculation-drainage & + & Liquid & Avg. & 2622 & 1153 & 134 & 1654 & 242 & 344 & 6150 & $0 \cdot 15$ & 5 & 5 & 1 \\
\hline Flocculation-drainage & + & Liquid & SD & & & & & & & & & (2) & (4) & (5) \\
\hline Flocculation-drainage & + & Fibre & Avg. & 4323 & 1888 & 217 & 2862 & 403 & 598 & 10291 & $0 \cdot 21$ & 107 & 202 & 141 \\
\hline Flocculation-drainage & + & Fibre & SD & & & & & & & & & (7) & (7) & (10) \\
\hline Decanter centrifuge & & Liquid & Avg. & 10449 & 3894 & 972 & 3010 & 1324 & 433 & 20081 & $0 \cdot 01$ & 12 & $0 \cdot 6$ & 1 \\
\hline Decanter centrifuge & & Liquid & SD & & & & & & & & & (2) & (6) & (5) \\
\hline Decanter centrifuge & & Fibre & Avg. & 8750 & 2589 & 580 & 894 & 816 & 246 & 13874 & $0 \cdot 00$ & 123 & 282 & 261 \\
\hline Decanter centrifuge & & Fibre & SD & & & & & & & & & (2) & (15) & (5) \\
\hline Decanter centrifuge & + & Liquid & Avg. & 5141 & 2275 & 269 & 3316 & 490 & 708 & 12199 & $0 \cdot 05$ & 4 & $<0 \cdot 1$ & 7 \\
\hline Decanter centrifuge & + & Liquid & SD & & & & & & & & & (2) & & (5) \\
\hline Decanter centrifuge & + & Fibre & Avg. & 4354 & 1778 & 207 & 2646 & 381 & 567 & 9933 & $0 \cdot 12$ & 135 & 302 & 229 \\
\hline Decanter centrifuge & + & Fibre & SD & & & & & & & & & $(0 \cdot 3)$ & (9) & (5) \\
\hline
\end{tabular}

* Avg.: average. 
Table 6. Concentration and speciation of sulphur and phosphorus components in the slurry and the solid and liquid fractions ( $S D$ in parentheses, $n=2$ )

\begin{tabular}{|c|c|c|c|c|c|c|c|c|c|c|}
\hline \multirow[b]{2}{*}{ Treatment } & \multirow[b]{2}{*}{ Acid } & \multirow[b]{2}{*}{$\begin{array}{l}\text { Manure } \\
\text { fraction }\end{array}$} & \multicolumn{2}{|c|}{ Total P } & \multicolumn{2}{|c|}{ Ortho-P } & \multicolumn{2}{|c|}{ S total } & \multicolumn{2}{|c|}{$\mathrm{H}_{2} \mathrm{~S}$ total } \\
\hline & & & $\begin{array}{l}\text { Avg. } \\
(\mathrm{g} / \mathrm{kg})\end{array}$ & (SD) & $\begin{array}{l}\text { Avg. } \\
\text { (g/kg) }\end{array}$ & SD & $\begin{array}{l}\text { Avg. } \\
(\mathrm{g} / \mathrm{kg})\end{array}$ & SD & $\begin{array}{l}\text { Avg. } \\
(\mathrm{mg} / \mathrm{kg})\end{array}$ & SD \\
\hline Untreated & & Slurry & $1 \cdot 9$ & $(0 \cdot 00)$ & $0 \cdot 2$ & $(0 \cdot 01)$ & $0 \cdot 6$ & $(0 \cdot 00)$ & & \\
\hline Untreated & + & Slurry & $1 \cdot 4$ & $(0 \cdot 07)$ & $0 \cdot 4$ & $(0 \cdot 02)$ & $4 \cdot 5$ & $(0 \cdot 07)$ & & \\
\hline Screw press & & Liquid & $2 \cdot 0$ & $(0 \cdot 07)$ & $0 \cdot 2$ & $(0 \cdot 03)$ & $0 \cdot 5$ & $(0 \cdot 01)$ & $0 \cdot 174$ & $(0 \cdot 023$ \\
\hline Screw press & & Solid & $2 \cdot 4$ & $(0 \cdot 07)$ & $0 \cdot 1$ & $(0 \cdot 02)$ & $1 \cdot 1$ & $(0 \cdot 00)$ & & \\
\hline Screw press & + & Liquid & $1 \cdot 4$ & $(0 \cdot 00)$ & $0 \cdot 4$ & $(0 \cdot 06)$ & $4 \cdot 3$ & $(0 \cdot 64)$ & $0 \cdot 002$ & $(0 \cdot 002)$ \\
\hline Screw press & + & Solid & $1 \cdot 5$ & $(0 \cdot 07)$ & $0 \cdot 4$ & $(0 \cdot 01)$ & $4 \cdot 0$ & $(0 \cdot 14)$ & $0 \cdot 001$ & $(0 \cdot 001)$ \\
\hline Flocculation-drainage & & Liquid & $0 \cdot 2$ & $(0 \cdot 00)$ & $0 \cdot 1$ & $(0 \cdot 01)$ & $0 \cdot 1$ & $(0 \cdot 01)$ & $<0 \cdot 001$ & \\
\hline Flocculation-drainage & & Solid & $2 \cdot 5$ & $(0 \cdot 00)$ & $0 \cdot 1$ & $(0 \cdot 01)$ & $0 \cdot 7$ & $(0 \cdot 03)$ & $0 \cdot 030$ & $(0 \cdot 024)$ \\
\hline Flocculation-drainage & + & Liquid & $0 \cdot 6$ & $(0 \cdot 01)$ & $0 \cdot 2$ & $(0 \cdot 01)$ & $2 \cdot 4$ & $(0 \cdot 07)$ & $<0 \cdot 001$ & \\
\hline Flocculation - drainage & + & Solid & $1 \cdot 4$ & $(0 \cdot 00)$ & $0 \cdot 4$ & $(0 \cdot 01)$ & $3 \cdot 7$ & $(0 \cdot 07)$ & $0 \cdot 001$ & $(0 \cdot 001)$ \\
\hline Decanter centrifuge & & Liquid & $0 \cdot 5$ & $(0 \cdot 00)$ & $0 \cdot 1$ & $(0 \cdot 00)$ & $0 \cdot 5$ & $(0 \cdot 01)$ & & \\
\hline Decanter centrifuge & & Solid & $7 \cdot 3$ & $(0 \cdot 64)$ & $0 \cdot 2$ & $(0 \cdot 00)$ & $1 \cdot 6$ & $(0 \cdot 00)$ & $0 \cdot 009$ & $(0 \cdot 001)$ \\
\hline Decanter centrifuge & + & Liquid & $1 \cdot 2$ & $(0 \cdot 00)$ & $0 \cdot 4$ & $(0 \cdot 03)$ & $4 \cdot 5$ & $(0 \cdot 07)$ & $<0 \cdot 000$ & \\
\hline Decanter centrifuge & + & Solid & $2 \cdot 5$ & $(0 \cdot 07)$ & $0 \cdot 3$ & $(0 \cdot 04)$ & $3 \cdot 9$ & $(0 \cdot 14)$ & $<0 \cdot 000$ & \\
\hline
\end{tabular}

P: phosphorus; S: sulphur; $\mathrm{H}_{2} \mathrm{~S}$ : hydrogen sulphide; Avg.: average.
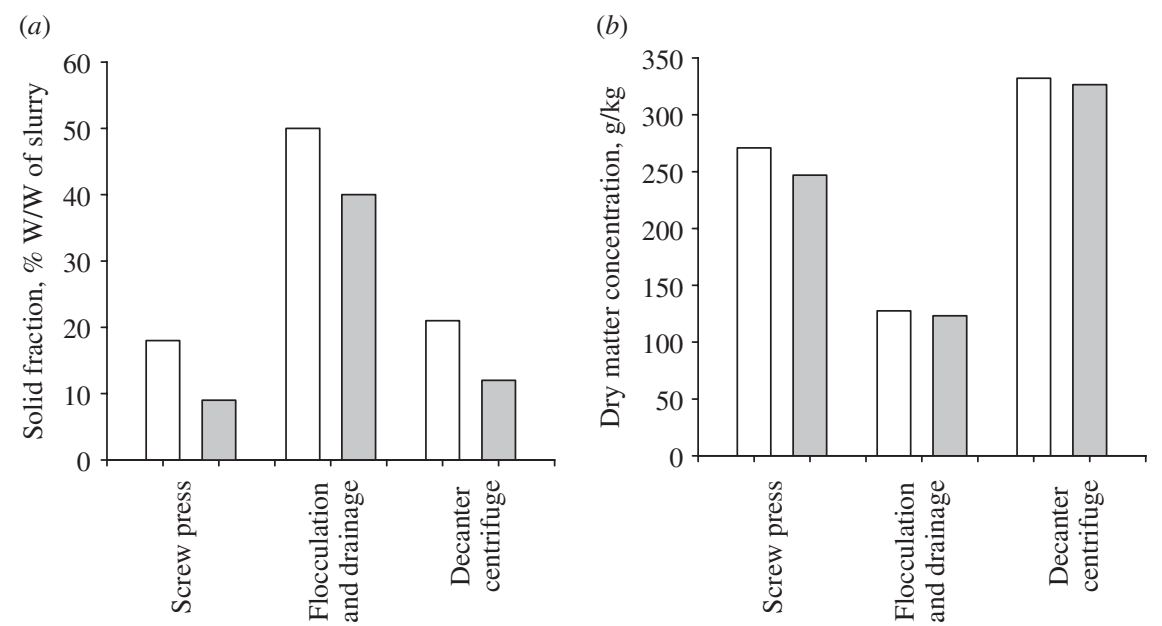

Fig. 1. Separation of slurry as affected by separation technology and the acidification of slurry: (a) the amount of solid manure fraction as a fraction of slurry treated and $(b)$ DM concentration in solid manure fraction. Untreated $(\square)$ and acidified fractions ( $\square$ ).

showed a higher BMP/kg VS. The BMP/kg VS of the other solid fractions, which were all similar, were not significantly different from those of acidified slurry or the liquid fraction from the screw press and decanter centrifuge.

The amount and composition of slurry and slurry fractions applied to fields were assessed using the conditions typical for a traditional Danish manure management system (Tables 1 and 2). First, when considering fulfilling the crop need by fertilizerefficient $\mathrm{N}$ alone, with the exception of the liquid fraction from flocculation and filtration, the acreage of land required was higher when applying treated fractions than the acreage needed when applying slurry (Fig. 3). Separation with flocculation and drainage transfers a large amount of $\mathrm{N}$ to the solid fraction. Therefore, the $\mathrm{N}$ supply in the liquid fraction from drainage covers the $\mathrm{N}$ requirement of a smaller winter wheat area. Acidification increased the field acreage of wheat where the $\mathrm{N}$ need could be fulfilled. Instead of looking solely at the fulfilment of the $\mathrm{N}$ requirement with manure application, calculations 
(a)

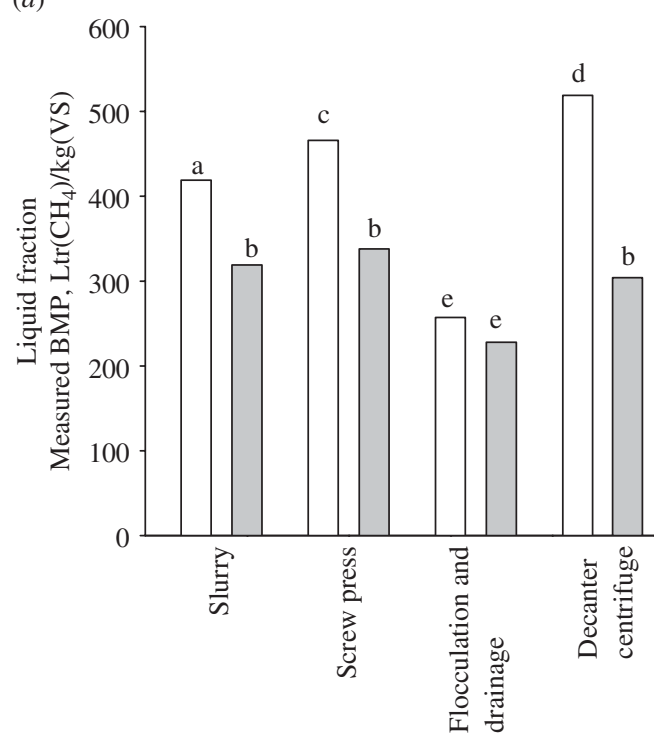

(b)

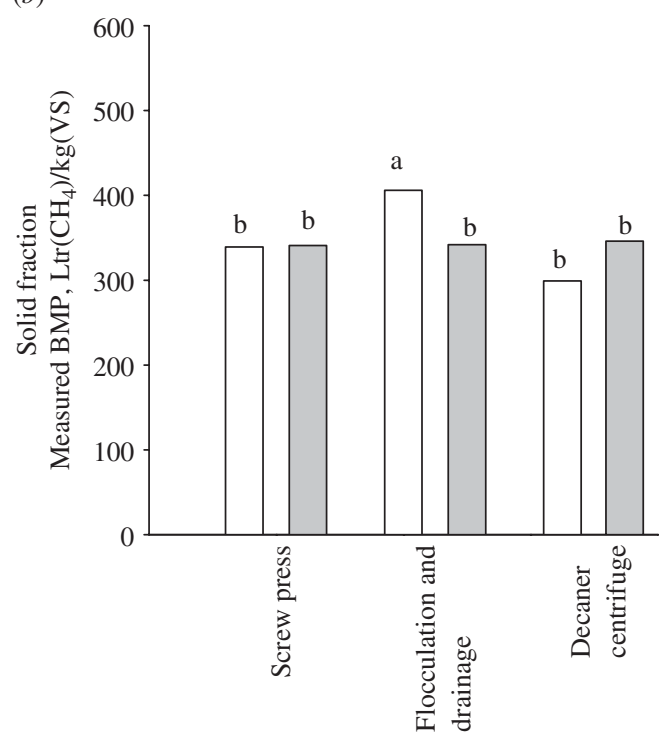

Fig. 2. Biogas production potential: (a) measured BMP of slurry and liquid fraction and $(b)$ measured BMP of solid fraction. Bars with different letters are significantly different $(P<0 \cdot 05)$. Untreated $(\square)$ and acidified fractions $(\square)$.

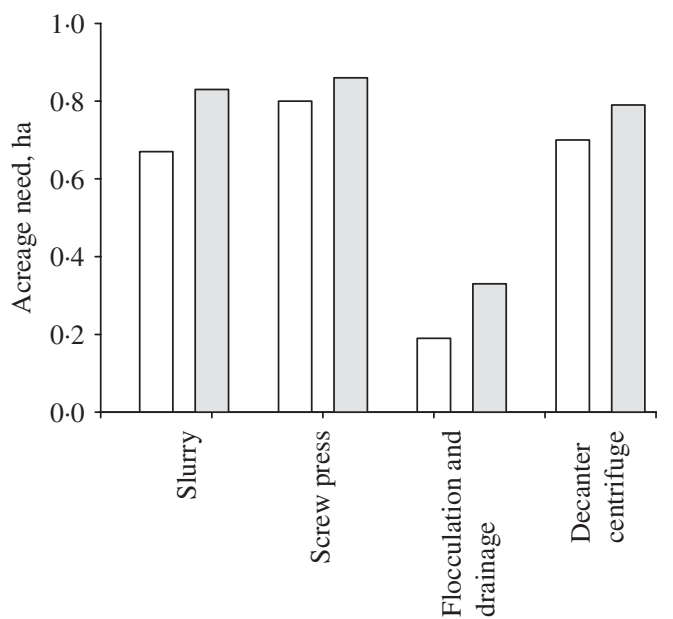

Fig. 3. The field area needed for spreading $30 t$ of slurry or the liquid manure fraction obtained from separating $30 \mathrm{t}$ of slurry to fulfil winter wheat requirements for available nitrogen (adjusted to MFE). Untreated ( $\square$ ) and acidified fractions ( $\square)$.

were made to establish the largest acreage of crop that could be fulfilled by nutrients $\mathrm{N}, \mathrm{P}$ or $\mathrm{K}$ using the lowest application rates of liquid fraction to fulfil cropland need (Fig. 4(a)). Potassium had the largest land demand when applying the non-acidified liquid fraction from flocculation and drainage and from decanter centrifugation to wheat. For the other uses of liquid fractions, $\mathrm{P}$ application demanded most land. The field area needed to spread the liquid and solid fraction to meet NPK requirements was, for most treatments, limited by P (Fig. 4(b)). The two exceptions were flocculation/drainage and centrifuge-treated slurry, where $\mathrm{K}$ limited the demand for land. The additional application of manure to meet the crop's $\mathrm{N}$ requirement would cause an oversupply of $\mathrm{P}$ and $\mathrm{K}$. Hence, mineral $\mathrm{N}$ fertilizer should be supplemented (Figs 4(c) and $(d)$ ). Acidification reduced the area with wheat needed, probably because, firstly, total $P$ in the whole slurry and liquid manure from the screw press was not affected by acidification and, secondly, acidification did not change the total $\mathrm{K}$ content in the liquid fraction of manure from either flocculation/ drainage or decanter centrifugation. In the case where both liquid and solid fractions were applied to fields, acidification reduced the area of land required and the amount of mineral fertilizers that needed to be added as well (Figs $4(b)$ and $(d)$ ). The reason for this was that more $\mathrm{N}, \mathrm{P}$ and $\mathrm{K}$ were recovered in the nonacidified solid fractions than in the acidified fractions.

In the current study, the concentration of $\mathrm{Zn}$ was $200-1610 \mathrm{mg} / \mathrm{kg}$ DM and Cu concentration was 50-174 mg/kg DM. The highest heavy metal concentrations were found in the solid fractions after separation by flocculation/drainage and decanter centrifuge (Table 4). After screw-press separation, however, slightly higher $\mathrm{Cu}$ and $\mathrm{Zn}$ concentrations were found in the liquid fraction than in the solid fraction. The concentration of $\mathrm{Zn}$ and $\mathrm{Cu}$ was higher in the solid fraction separated using coagulants than in the slurry before the separation treatment. 
(a)

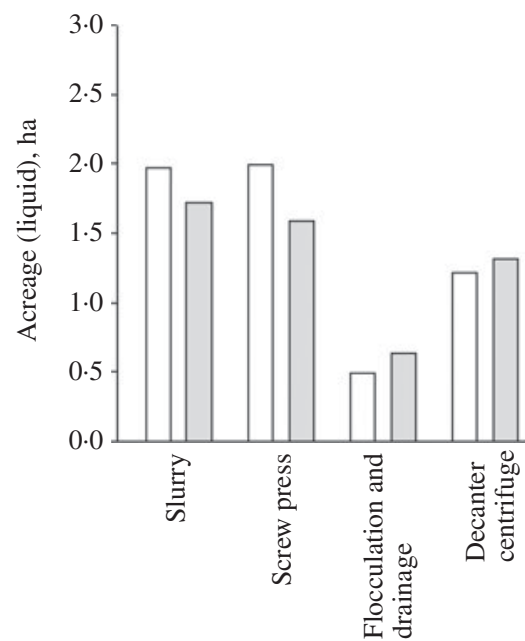

(c)

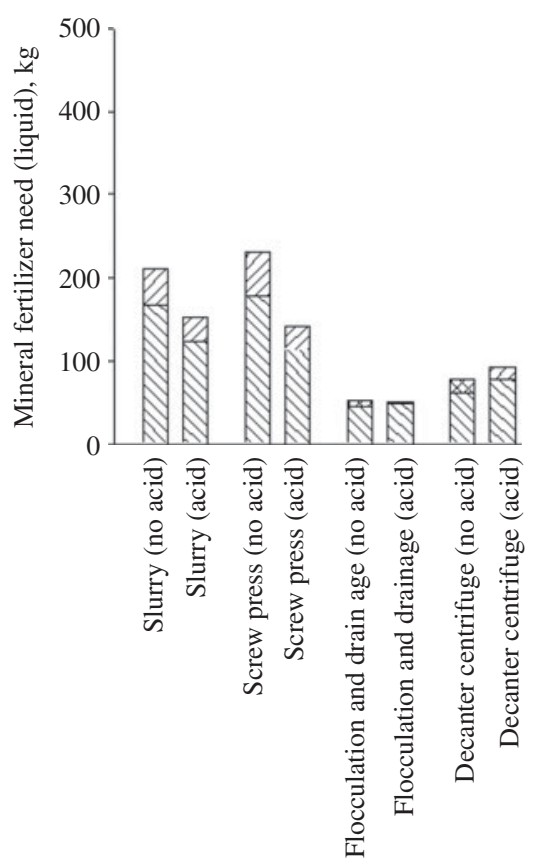

(b)

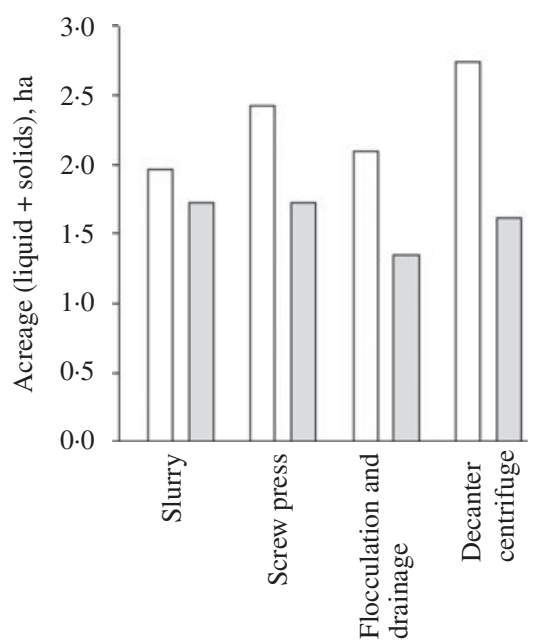

(d)

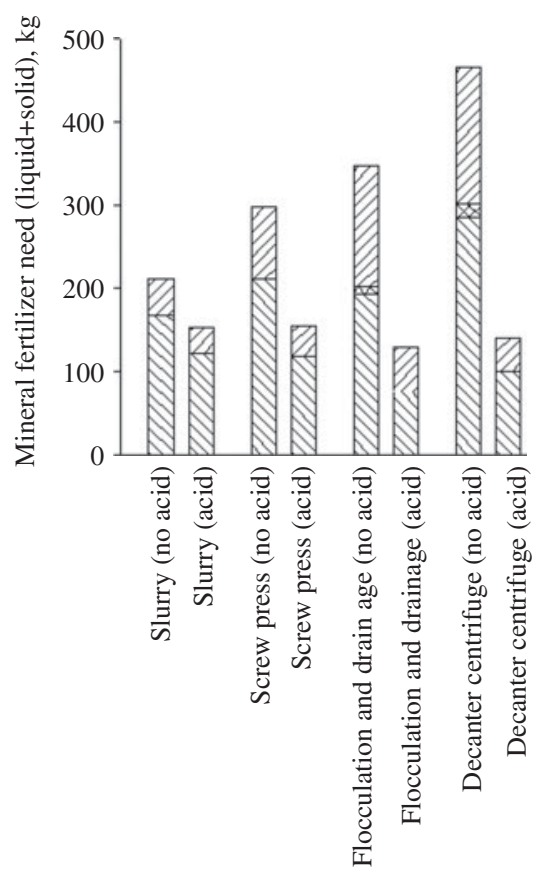

Fig. 4. (a) The field area to which liquid manure has to be spread to supply winter wheat with the nutrients that, at the lowest manure application rate, fulfils crop requirements. (b) The total area needed for application of liquid fraction to winter wheat and solid fraction to maize to supply them with the nutrients that, at the lowest manure application rate, fulfils crop requirements. Untreated ( $\longleftarrow$ ) and acidified fractions ( $\square$ ). (c) Additional mineral fertilizer needed to fulfil the wheat crop demand, where only liquid manure was applied. $(d)$ Additional mineral fertilizer needed to fulfil the crop demand where liquid fraction was applied to a wheat crop and solid manure fraction was applied to a maize crop. Nitrogen ( phosphorus (

Liquid fraction application to wheat fields did not increase the Cu content of soil except for wheat fields treated with the non-acidified liquid fraction from decanter centrifugation. However, in maize fields on which solid fractions from pig slurry separation were applied, the content of Cu did increase (Fig. 5). With the exception of wheat fields on which flocculated and drained liquid was applied, more Zn would be applied than removed with grain and straw from wheat and maize fields (Fig. 5).

The cost of separation varied greatly between the three separation technologies, whereas the cost of application was not particularly affected by separation (Fig. 6). When including fixed and operational costs 

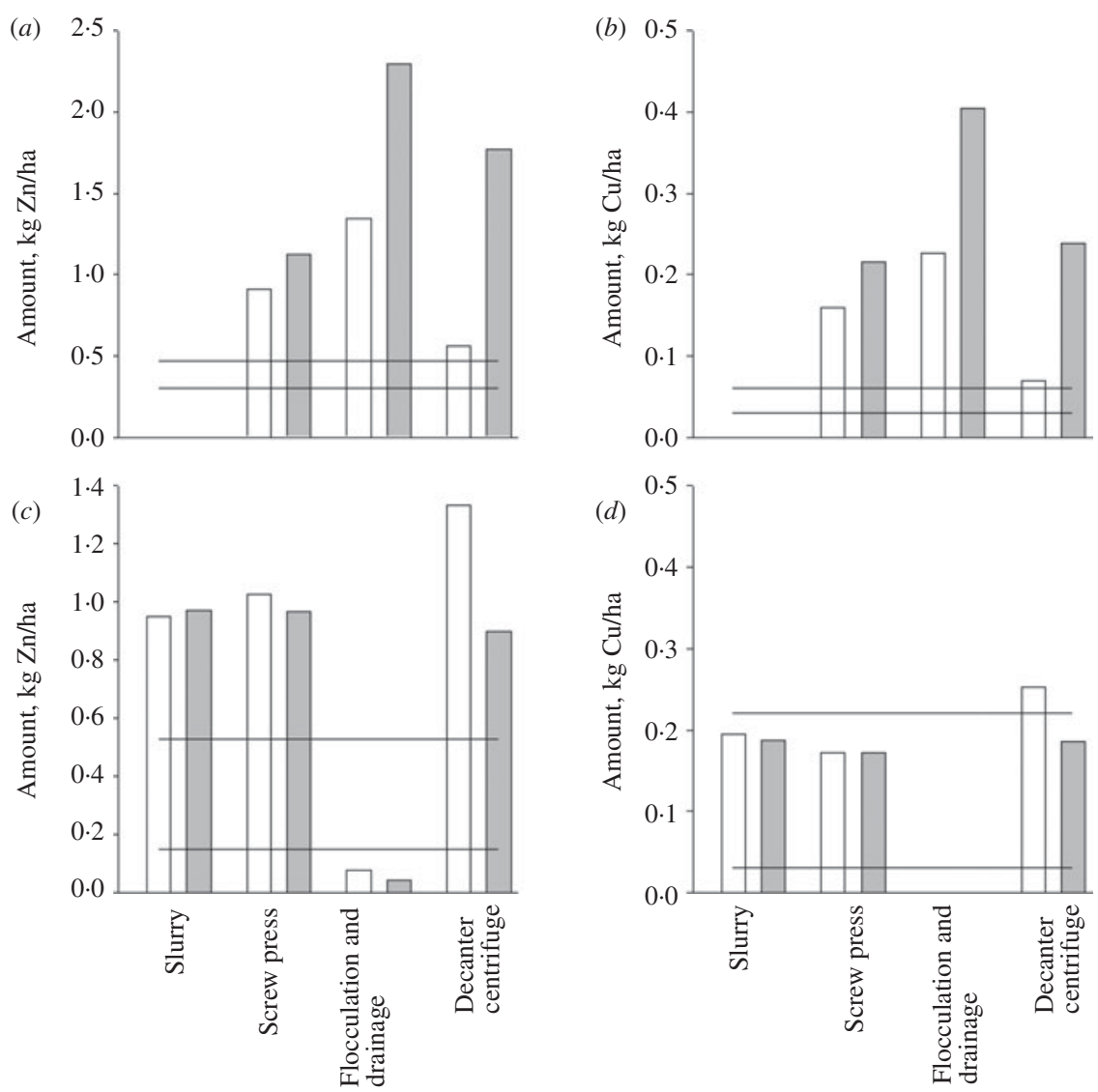

Fig. 5. Amounts of zinc $(\mathrm{Zn})$ and copper $(\mathrm{Cu})$ added per hectare of agricultural land at optimal fertilization with nitrogen. $(a, b) \mathrm{Zn}$ and $\mathrm{Cu}$, respectively, added in solids to maize fields. (c, d) $\mathrm{Zn}$ and $\mathrm{Cu}$, respectively, added in liquids to wheat fields. The horizontal lines indicate estimated minimum and maximum removal of $\mathrm{Zn}$ and maximum removal of $\mathrm{Cu}$ with the straw and grain in the harvested crop. Untreated fractions $(\square)$ and acidified fractions ( $\square$ ).

(Table 7), the cost of acidification amounted to $5 \cdot 2 \mathrm{US} \$ / \mathrm{m}^{3}$ for a pig farm with 392 livestock units producing $6899 \mathrm{t}$ of slurry annually, almost doubling the management cost of slurry (Fig. 6). The MFE increased from 46 to $81 \mathrm{~kg}$ mineral $\mathrm{N}$ per $100 \mathrm{~kg} \mathrm{~N}$ managed. At excretion, $1 \mathrm{t}$ of slurry contains C. $7 \mathrm{~kg}$ N. Acidification of the slurry on application contributed to an increase in MFE of c. $2 \cdot 5 /$ t slurry, corresponding to a cost saving on fertilizers of US $\$ 2 /$ slurry.

Separation costs differed, with the screw press being the least expensive: flocculation costs were $70 \%$ higher than screw-press separation and decanter centrifugation 98\% higher (Fig. 6). Application costs, on the other hand, showed no significant variation between the different separation techniques, including the base scenario involving no separation. Therefore, transporting and the application of two manure fractions to a field is only slightly more expensive than transporting and applying one fraction (Fig. 6).

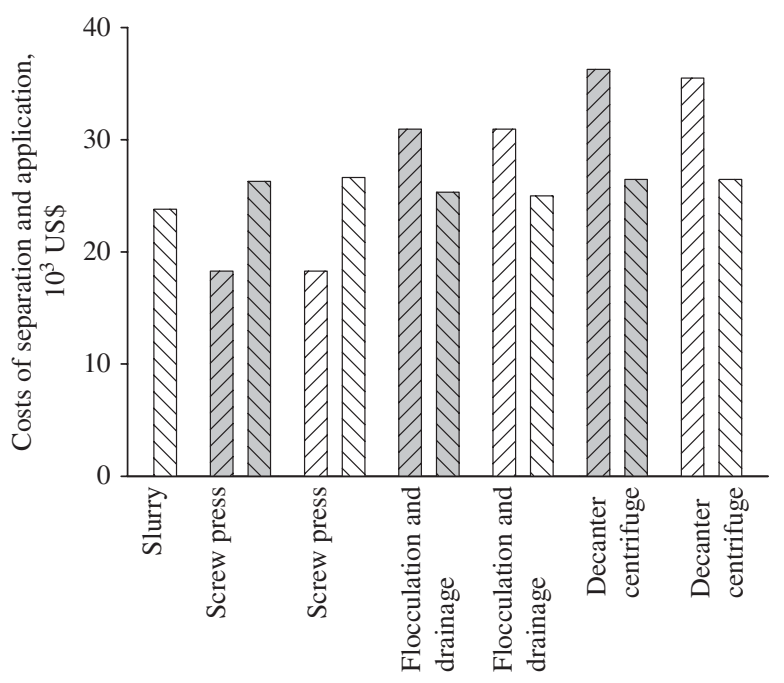

Fig. 6. Total costs of separation and application in US\$. Separation and untreated ( $\square \longrightarrow$ ), separation and acidified $(\square \backslash \nabla)$, application and untreated ( $\square \backslash \checkmark)$ and application and acidified ( $\Delta \backslash \nabla)$. 
Table 7. Costs of acidification

\begin{tabular}{lll}
\hline \hline Parameter* & Unit & $\begin{array}{l}\text { Costs for acid tank, } \\
\text { process tank, mounting, etc. }\end{array}$ \\
\hline Investment & $\cup S \$$ & 222000 \\
Annual capital costs & $\cup S \$ / y r$ & 19689 \\
Net labour input & $\cup S \$ / y r$ & 956 \\
Maintenance/service contract & $\cup S \$ / y r$ & 4901 \\
Electricity & $\cup S \$ / \mathrm{m}^{3}$ & $0 \cdot 37$ \\
Acid & $\cup S \$ \mathrm{~m}^{3}$ & $1 \cdot 16$ \\
\hline \hline
\end{tabular}

* Annual costs based on a 15-year depreciation period and an interest rate of $4 \%$. Net labour input $(0 \cdot 01$ man hour $/ \mathrm{t})$, service contract costs, extra electricity consumption $(3 \mathrm{kWh} / \mathrm{t})$ and acid consumption $(6 \cdot 1 \mathrm{~kg} / \mathrm{t})$ and cost (US\$0.19/t) (Environmental Protection Agency 2011).

\section{DISCUSSION}

The current study provides an assessment of the effects of acidification and separation of slurry from a pig fattening farm on the characteristics of inorganic manure components, organic components and biogas production, land requirement for the field application of manure, heavy metal loading and costs related to the treatments.

\section{Manure characteristics}

The concentration of the inorganic components was higher than the concentration measured in the 1995 study of Sommer \& Husted (1995). This was probably due to better water management at this farm, which is part of a research station where the addition of spilled drinking water and excess washing water to the slurry is reduced. The lower water content will not greatly affect separation efficiencies related to the physical and chemical characteristics of DM, but it will reduce the volume of slurry that has to be treated (Hjorth et al. 2010).

The VS of the liquid fraction contained a lower fraction of lignin than the solid fraction, and was within the range measured in earlier studies of manure from fattening pig production (Bruni et al. 2010; Triolo et al. 2011).

The TIC of untreated slurry was at the same level, whereas in acid-treated slurry it was $0 \cdot 1$ times the concentration reported in the study of Sommer \& Husted (1995). The lower TIC and VFA in the acidtreated slurry compared to the untreated slurry was due to the inhibition of microbial transformation of the organic matter caused by acidification to $\mathrm{pH} 5 \cdot 5$ (Petersen et al. 2012). Consequently, the VFA in acidified slurry primarily consisted of the VFA in faeces excreted by the animals, whereas the concentration of VFA in untreated slurry was about one-third higher due to the production of VFA during storage (Møller et al. 2004b).

The concentration of $\mathrm{H}_{2} \mathrm{~S}$ was not affected by the addition of sulphuric acid, indicating that sulphate was not reduced in the acidified slurry. Ortho-P was higher in the acidified slurry than in the untreated slurry, indicating that more $\mathrm{P}$ was dissolved at a lower pH (Hjorth et al. 2010).

Organic components and biogas production potential

The reduced total amount of DM in the solid fraction from separation of acidified slurry indicates that lowering the $\mathrm{pH}$ of the slurry changes the particle size, the charges of colloid particles and inorganic components, and/or the amount of dissolved compounds.

The BMP of the slurry and liquid fractions was lowered by the acidification pre-treatment, which is in accordance with a study by Sutaryo et al. (2013). However, acidification only lowered the BMP of the solid fraction produced by flocculation and drainage which had a large liquid content. Hence, the process inhibitor appears to be located in the liquid; the inhibitor could therefore be $\mathrm{H}_{2} \mathrm{~S}$ (Moset et al. 2012). Lignin is an inhibitor of VS transformation to biogas (Triolo et al. 2013), therefore the liquid fraction with a low lignin content showed a higher BMP than the solid fraction. The BMP of the non-acidified liquid fraction was high, which must be due to the low lignin and carbohydrate concentration of this fraction. Furthermore, the BMP of the liquid fraction from the flocculation and drainage experiment showed the lowest BMP per $\mathrm{kg}$ of VS because the 
treatment removed the organic matter with degradable components following the efficient removal of particles. The BMP of the solid fractions produced by flocculation and drainage was slightly higher than the BMP of the other solid fractions, indicating that, in general, the VS transferred to this fraction was of a similar composition.

A low $\mathrm{pH}$ and high sulphur (S) content inhibits the anaerobic fermentation of the biomass and causes a low BMP in the liquid fractions, but not in the solids. Thus, if an operator uses acidified biomass, it has to be blended with biomass with a high buffer capacity, neutral $\mathrm{pH}$ and low $\mathrm{S}$ content in order to maintain the $\mathrm{pH}$ of the digestate and to keep the $\mathrm{S}$ concentration low.

\section{Field acreage need and crop nutrient requirement}

Separation facilitates the removal of $N$ from a farm with excess nitrogen. By only considering $\mathrm{N}$ application, with the exception of flocculation and drainage separation, the separation increases the wheat acreage to which the slurry and liquid fraction is applied to cover the wheat crop need efficiently. The area needed to spread the liquid fraction from decanter and screwpress separation to meet plant $\mathrm{N}$ requirements in full was a little higher for the liquid manure than for the slurry because the MFE was much higher for the liquid manure fraction than for the slurry. However, the current study shows that efficient use of manure $\mathrm{N}, \mathrm{P}$ and $\mathrm{K}$ often requires an equivalent or larger area of agricultural land than with the use of untreated slurry. On farms where acidified slurry and slurry separation products are produced and spread, separation, except with the screw press, reduced the need for land. Separation slightly enhanced the efficient use of the nutrients from acidified slurry. Consequently, the reduction in the land area required was due to a combination of a better match between crop NPK requirements and the NPK applied with the manure. Applying liquid manure from flocculation/drainage and decanter centrifugation largely avoided the oversupply of $\mathrm{P}$ and $\mathrm{K}$, and hence reduced the need for additional mineral fertilizer on wheat crops.

The MFE was assumed to be similar for the three solid fraction products produced in the current study, but tests may show that the solid fraction from flocculation and drainage with a high total ammoniacal nitrogen (TAN $=\mathrm{NH}_{3}+\mathrm{NH}_{4}^{+}$) concentration has a higher MFE than the solid fractions produced by the screw-press treatment or by centrifugation.
Heavy metals

Zinc ( $\mathrm{Zn}$ ) and copper (Cu) are often added to pig feed to reduce disease, therefore the concentration of these metals in pig slurry is high compared with the concentration in slurry from, for example, cattle (Møller et al. 2007). The $\mathrm{Zn}$ concentrations were similar to those measured in earlier studies characterizing pig slurries, and $\mathrm{Cu}$ concentrations were much lower than in the study of Møller et al. (2007), for example, where the $\mathrm{Zn}$ concentration was 770$911 \mathrm{mg} /(\mathrm{kg} \mathrm{DM})$ and Cu was 494-640 mg/(kg DM). Slurry separation using coagulants and additives before drainage and by decanter centrifuge retains more of the small particles in the solid fractions (Peters et al. 2011) which have high $\mathrm{Zn}$ and $\mathrm{Cu}$ concentrations (Popovic et al. 2012). Nevertheless, the concentrations of $\mathrm{Cu}$ and $\mathrm{Zn}$ in the slurry and slurry fractions were below the Danish limits set for the spreading of sewage sludge to agricultural fields, which are 4 and $1 \mathrm{~g} / \mathrm{kg} \mathrm{DM}$ for $\mathrm{Zn}$ and $\mathrm{Cu}$, respectively (Anonymous 2006).

Therefore, the addition of pig slurry to fields will increase the concentration of $\mathrm{Cu}$ and $\mathrm{Zn}$ in the soil. The slurry and liquid fraction will not increase $\mathrm{Cu}$ in wheat fields except for a slight increase when the liquid fraction from the decanter centrifuge treatment is applied. Addition of solid fractions to maize fields will increase both the $\mathrm{Zn}$ and $\mathrm{Cu}$ content of the soil. However, plant availability of trace elements such as $\mathrm{Cu}$ and $\mathrm{Zn}$ is affected by soil properties such as texture and especially $\mathrm{pH}$ : slightly acidic soil $\mathrm{pH}$ favours the uptake of $\mathrm{Cu}$ and $\mathrm{Zn}$ compared with alkaline soil $\mathrm{pH}$ (Cabral et al. 1998). As far as it is known, the effect of slurry acidification before separation on the plant uptake of heavy metals from the applied separated fractions has not yet been investigated, but it is likely that the bioavailability of $\mathrm{Cu}$ and $\mathrm{Zn}$ from the acidified fractions will be comparatively high, or higher than the values found in the literature.

\section{Cost estimates}

The automated slurry acidification system reduces labour costs related to the removal of slurry from the animal house, while the cost of covering slurry stores is not affected and the application costs is reduced for slurry applied to grassland. However, there are also indications that an increased need for liming should be expected (Petersen et al. 2011).

It should be noted that, in the current study, unit costs are based on the norms of contractors who are 
hired by most Danish farmers to apply manure to fields, thus eliminating the additional costs of needing to acquire two manure application machines. Therefore, separation does not increase application costs. In the case of farmer-owned machines, the influence of using two machine systems (solid manure application and slurry application) could increase application costs, especially if small amounts of manure are to be handled annually. Acidification and separation are expensive treatments and the associated costs in most cases will not be matched by a more efficient use of the manure.

\section{CONCLUSIONS}

The current study shows that the separation of manure contributes to a more efficient use of the nutrients in animal slurry if its application is adjusted to the NPK needs of the plants. The consequence of using this strategy was that separation will not reduce the area of land to which slurry must be applied, as would be the case if the focus had been on $\mathrm{N}$ application alone and an oversupply of $\mathrm{P}$ and $\mathrm{K}$ were not considered problematic. Acidification reduced the need for supplementary fertilizers. There was an accumulation of heavy metals in fields receiving slurry and slurry separation products, with the exception of copper accumulation in wheat fields where the liquid fraction or slurry applied. The costs of the different separation techniques varied between 2.65 and $5.26 \mathrm{US} \$ / \mathrm{m}^{3}$, with the screw-press method being the least costly and the decanter method the most costly. The application of the separated manure products ranged between 3.45 and $3.85 \cup S \$ / \mathrm{m}^{3}$, showing a relatively constant cost level for the different application methods.

The authors wish to acknowledge the support given by the Danish Council for Strategic Research (DSF) under the 'Strategic Research in Sustainable Energy and Environment' research programme to the project 'Clean and environmentally-friendly animal waste technologies for fertilizer and energy production (Cleanwaste)'. We also thank Rikke Jensen for her support during the experiments.

\section{REFERENCES}

Anonymous (2006). Bekendtgørelse om anvendelse af affald til jordbrugsformål (Slambekendtgørelsen) (Ministerial Order for Application of Organic Waste to Agricultural Land). Copenhagen, Denmark: Miljøministeriet (Ministry of Environment.
Anonymous (2007). Soil improvers and growing media: sample preparation for chemical and physical tests, determination of dry matter content, moisture content and laboratory compacted bulk density. European Standard CSN EN 13040. Brussels, Belgium: European Standards.

APHA (2005). Standard Methods for the Examination of Water and Wastewater, 21st edn. Washington, DC: American Public Health Association.

Berenguer, P., Cela, S., Santiveri, F., Boixadera, J. \& Lloveras, J. (2008). Copper and zinc soil accumulation and plant concentration in irrigated maize fertilized with liquid swine manure. Agronomy Journal 100, 1056-1061.

BiRkMOSE, T. (2009). Nitrogen Recovery from Organic Manures: Improved Slurry Application Techniques and Treatment-The Danish Scenario. Proceedings of the International Fertiliser Society No. 656. York, UK: International Fertiliser Society.

Bruni, E., Jensen, A. P. \& AngelidAkI, I. (2010). Steam treatment of digested biofibers for increasing biogas production. Bioresource Technology 101, 7668-7671.

Cabral, F., Vasconcelos, E. \& Cordovil, C. M. D. S. (1998). Effects of solid phase from pig slurry on iron, copper, zinc, and manganese content in soil and wheat plants. Journal of Plant Nutrition 21, 1955-1966.

EC (2009). Commission Regulation (EC) No. 152/2009 of 27 January 2009 laying down the methods of sampling and analysis for the official control of feed. Official Journal of the European Union L54, 1-130.

Eriksen, J., Adamsen, A. P. S., Norgatrd, J. V., Poulsen, H. D., Jensen, B. B. \& Petersen, S. O. (2010). Emissions of sulfur-containing odorants, ammonia, and methane from pig slurry: effects of dietary methionine and benzoic acid. Journal of Environmental Quality 39, 1097-1107.

Environmental Protection Agency (2010a). Separering af Gylle med Skruepresse (Separation of Slurry using Screw Press), version 1, 2010. Copenhagen, Denmark: The Danish Environmental Protection Agency (in Danish). Available from: http://www2.mst.dk/Wiki/(X(1)S(ik5ihnes vnlxd02ccob45xqw))/GetFile.aspx?File=/BAT/Teknologi blade/Gyllesepareringskruepresser.pdf (assessed March 2014).

Environmental Protection Agency (2010b). Separering af Gylle med Kemisk Fældning (Separation of Slurry using Chemical Extraction), version 1, 2010. Copenhagen, Denmark: The Danish Environmental Protection Agency (in Danish). Available from: http://mst.dk/media/mst/66920/ Gylleseparering\%20-\%20kemisk\%20f\%C3\%A6ldning.pdf (accessed March 2014).

Environmental Protection Agency (2010c). Separering af Gylle med Dekanter-Centrifuge (Separation of Slurry using Decanter Centrifuge), version 1, 2010. Copenhagen, Denmark: The Danish Environmental Protection Agency (in Danish). Available from: http://www2.mst.dk/Wiki/ (X(1)S(ik5ihnesvnlxd02ccob45xqw))/GetFile.aspx?File=/BAT/ Teknologiblade/Gyllesepareringdekantercentrifuger.pdf (accessed March 2014).

Environmental Protection Agency (2011). Forudsætninger for de Økonomiske Beregninger ved Forsuring 
(Prerequisites for Economic Calculations Related to Acidification). Analysis by Consultancy Company NIRAS Inc. Copenhagen, Denmark: NIRAS (in Danish). Available from: http://mst.dk/media/mst/66907/foruds $\%$ C3\%A6tninger\%20bat\%20juni\%202010\%20forsuring \%20KS.pdf (accessed March 2014).

Farmtal Online (2013). Knowledge Centre for Agriculture. Aarhus, Denmark: Danish Agricultural Advisory Service (DAAS). Available from: https://farmtalonline.dlbr.dk/ Navigation/NavigationTree.aspx (accessed March 2014).

Goering, H. K. \& VAN Soest, P.J. (1970). Forage Fiber Analysis: Apparatus, Reagents, Procedures and Some Applications. Agriculture Handbook No. 379. Washington, DC: Agriculture Research Service, USDA.

Hansen, M. N., Henriksen, K. \& Sommer, S. G. (2006). Observations of production and emission of greenhouse gases and ammonia during storage of solids separated from pig slurry: effects of covering. Atmospheric Environment 40, 4172-4181.

Hansen, M. N., Sommer, S. G., Hutchings, N. J. \& Sørensen, P. (2008). Emissionsfaktorer til beregning af ammoniakfordampning ved lagring og udbringning af husdyrgødning (Emissions Factors for Calculation of Ammonia Volatilization by Storage and Application of Animal Manure). DJF Husdyrbrug nr. 84. Tjele, Denmark: Aarhus University.

Hjorth, M., Christensen, K. V., Christensen, M. L. \& Sommer, S. G. (2010). Solid-liquid separation of animal slurry in theory and practice: a review. Agronomy for Sustainable Development 30, 153-180.

JenSEN, L. S. (2013). Animal manure fertiliser value, crop utilisation and soil quality impacts. In Animal Manure Recycling: Treatment and Management. An Introductory Reference Book (Eds S. G. Sommer, M. L. Christensen, T. Schmidt \& L.S. Jensen), pp. 295-328. Chichester, UK: Wiley-Blackwell.

Kal, P., Pedersen, P., Jensen, J.E., Hansen, M. N. \& SOMmer, S. G. (2008). A whole-farm assessment of the efficacy of slurry acidification in reducing ammonia emissions. European Journal of Agronomy 28, 148-154.

Mertens, D. R. (2002). Gravimetric determination of amylase-treated neutral detergent fiber in feeds with refiuxing in beakers or crucibles: collaborative study. Journal of AOAC International 85, 1217-1240.

Moset, V., Cambra-López, M. \& Møller, H. B. (2012). The inhibiting effect of sulphate on thermophilic anaerobic digestion of cattle and pig waste slurry. Transactions of the American Society of Agricultural and Biological Engineers 55, 2309-2317.

MurPhy, J. \& RileY, J.P. (1962). A modified single solution method for the determination of phosphate in natural waters. Analytica Chimica Acta 27, 31-36.

Møleze, H. B., Sommer, S. G. \& Ahring, B. K. (2004a). Methane productivity of manure, straw and solid fractions of manure. Biomass \& Bioenergy 26, 485-495.

Møller, H. G., Sommer, S. G. \& Ahring, B. K. (2004b). Biological degradation and greenhouse gas emissions during pre-storage of liquid animal manure. Journal of Environmental Quality 33, 27-36.

Møller, H. B., Jensen, H.S., Tobiasen, L. \& Hansen, M. N. (2007). Heavy metal and phosphorus content of fractions from manure treatment and incineration. Environmental Technology 28, 1403-1418.

Peters, K., HJorth, M., Jensen, L. S. \& Magid, J. (2011). Carbon, nitrogen, and phosphorus distribution in particle sizefractionated separated pig and cattle slurry. Journal of Environmental Quality 40, 224-232.

Petersen, S. O., Nyord, T., Eriksen, J., Sørensen, P. \& ElsgaArd, L. (2011). Note on Acidification of Slurry for Plant Production. Aarhus, Denmark: Aarhus University.

Petersen, S. O., Andersen, A. J. \& Eriksen, J. (2012). Effects of cattle slurry acidification on ammonia and methane evolution during storage. Journal of Environmental Quality 41, 88-94.

Popovic, O., HJorth, M. \& Jensen, L. S. (2012). Phosphorus, copper and zinc in solid and liquid fractions from fullscale and laboratory-separated pig slurry. Environmental Technology 33, 2119-2131.

Sommer, S. G. (2001). Effect of composting on nutrient loss and nitrogen availability of cattle deep litter. European Journal of Agronomy 14, 123-133.

Sommer, S. G. \& Husted, S. (1995). The chemical buffer system in raw and digested animal slurry. Journal of Agricultural Science, Cambridge 124, 45-53.

Sørensen, C. G. \& Møller, H. B. (2006). Operational and economic modeling and optimization of mobile slurry separation. Applied Engineering in Agriculture 22, 185-193.

SøRENSEN, P. \& ERIKSEN, J. (2009). Effects of slurry acidification with sulfuric acid combined with aeration on the turnover and plant availability of nitrogen. Agriculture, Ecosystems \& Environment 131, 240-246.

Sutaryo, S., Ward, A. J. \& Møller, H. B. (2013). Anaerobic digestion of acidified slurry fractions derived from different solid-liquid separation methods. Bioresource Technology 130, 495-501.

Sutton, M. A., Oenema, O., Erisman, J. W., Leip, A., van GrinsVen, H. \& WinIWARTER, W. (2011). Too much of a good thing. Nature 472, 159-161.

Triolo, J. M., Sommer, S. G., Møller, H. B., Weisbjerg, M. R. \& JIANG, X. Y. (2011). A new algorithm to characterize biodegradability of biomass during anaerobic digestion: influence of lignin concentration on methane production potential. Bioresource Technology 102, 9395-9402.

Triolo, J. M., Ward, A. J., Pedersen, L. \& Sommer, S. G. (2013). Characteristics of animal slurry as a key biomass for biogas production in Denmark. In Biomass Now: Sustainable Growth and Use (Ed. M. D. Matovic), pp. 307-326. Rijeka, Croatia: InTech.

VAN SOEST, P.J. (1963). Use of detergents in the analysis of fibrous feeds. II. A rapid method for the determination of fiber and lignin. Journal of AOAC International 46, 829-835. 